\title{
La situación actual de la participación política de las mujeres de Tonalá, Puerto Morazán, 2016
}

\author{
Current situation in Politic participation of the Women from Tonalá, Puerto Morazán, 2016 \\ Carmen Isabel Hernández Rivera ${ }^{1}$ \\ Idania Mercedes Bolaños Cerda ${ }^{2}$ \\ Noelia Erlinda $\mathrm{Cea}^{3}$
}

\section{Resumen}

En los últimos años en Nicaragua se ha fomentado la participación de la mujer en todos los niveles de la sociedad, con el fin de que estas se sientan parte y se apropien también del desarrollo de las comunidades. En Nicaragua, el área en la que más se ha visto esta participación ha sido en el ámbito político, donde incluso se han reformado leyes que promueven la participación equitativa de las mujeres, en todos los procesos de elección política. Sin embargo, es relevante conocer, si estos objetivos planteados por el gobierno central, están llegando también a las mujeres de las comunidades rurales, si se ha promovido la participación femenina con el mismo auge que se promueve en las urbes; para ello se plantea conocer los índices de participación de las mujeres de la comunidad de Tonalá, a través de encuesta distribuidas por los 12 barrios de la comunidad y aplicadas a 193 mujeres, donde se comprobó la baja participación femenina a pesar de todas las políticas públicas que fomentan la misma.

Palabras clave: Participación; mujer; política; desarrollo; empoderamiento

\section{Abstract}

In the last years in Nicaragua, the participation of women in different society levels has been promoted, with the purpose of making them feel part and also take ownership in the development of their communities. In Nicaragua, the area where this participation has most been seen has been in the political sphere, where even laws that promote the equal participation of women have been reformed in all the processes of political choice. However, it is relevant to know, if these objectives set by the central government, are also reaching women in rural communities, if female participation has been promoted with the same boom that is promoted in the cities; For this, it is proposed to know the participation rates of women in Tonalá community, through a survey distributed by the 12 neighborhoods of the community and applied to 193

1 Master en Desarrollo Local Sostenible, Profesora de la Escuela de Ciencias Agrarias y Veterinarias de la Universidad Nacional Autónoma de Nicaragua-León. Correo: carmen.hernandez@ev.unanleon.edu.ni ORCID: https://orcid.org//oooo-0002-3002-5255

2 Master en Turismo y Desarrollo, Profesor de la Facultad de Ciencias Económicas y Empresariales de la Universidad Nacional Autónoma de Nicaragua-León. Correo: idania@ce.unanleon.edu.ni; No. ORCID: https://orcid.org//oooo-0oo1-5158-1050

3 Licenciada en Estadística, Profesor de la Escuela de Ciencias Agrarias y Veterinarias de la Universidad Nacional Autónoma de Nicaragua- León. Correo: noelia.cea@ev.unanleon.edu.ni; No. ORCID: https://orcid.org//oooo-ooo1-7913-9459

Recibido: 05/02/2018 Aprobado: 30/04/2018 
women, where the low female participation was verified despite all public policies that encourage it.

Keywords: Participation; woman; politics; development; empowerment

\section{Introducción}

Para el desarrollo de la sociedad nicaragüense la participación de las mujeres en sus diferentes ámbitos dentro de la comunidad es relevante. A pesar de ello, toda la contribución femenina ha sido visible en determinados momentos, manteniéndose por lo regular considerablemente aislada. La presencia de las mujeres siempre fue mucho mayor en la base del sistema social que en las cúspides políticas e institucionales.

La participación de las mujeres en la disputa por derrotar el absolutismo de los Somoza y el incremento importante de su presencia durante el agudo proceso sociopolítico de los años ochenta, si bien no cambió por completo la tendencia histórica de su reducida presencia en los espacios de toma de decisión, mejoró la presencia de las mismas originando un salto en la visibilidad de las mujeres (Rivas, Salgado, Narvaéz, \& León, 2015). El Gobierno de Reconciliación y Unidad Nacional (GRUN) desde 2007, ha venido creando y ejecutando leyes que promuevan la participación de las mujeres en los ámbitos políticos, sociales y comerciales, como la reforma realizada en la Ley 40, la que obliga a los partidos políticos a que el $50 \%$ de sus candidatos sean mujeres, o la creación de la Ley 648 que se fundamenta en la igualdad, equidad, justicia, no discriminación y no violencia, así como el respeto a la dignidad y la vida de las personas (Asamblea Nacional de Nicaragua, 2013).

Sin embargo, a pesar de la promoción e implementación de leyes que incentivan la inclusión y empoderamiento de la mujer, esto no es suficiente para el desarrollo de las mujeres en otras áreas. Por ello se realizó el estudio sobre el empoderamiento e inclusión de la mujer, en la comunidad de Tonalá Puerto Morazán, Chinandega, donde se evaluaron tres dimensiones del desarrollo sostenible que son la dimensión social, política y económica, con el fin de observar los avances en el empoderamiento e inclusión de las mujeres en la comunidad. Este fue un estudio descriptivo de corte transversal, dirigido tanto a hombres como mujeres económicamente activas entre los 20 y 40 años, para ellos se realizaron encuestas entrevistas a organizaciones públicas y privadas que tenían relación con el empoderamiento e inclusión de la mujer en la comunidad.

El empoderamiento e inclusión de la mujer es un tema de suma importancia si se desea potenciar el desarrollo local sostenible en cualquier territorio, no se puede pensar en desarrollo sin asegurarse que todos estén involucrados en el mismo y tomando en cuenta que el desarrollo local sostenible se puede entender como aquel proceso que permite la existencia de los recursos necesarios para el desarrollo de las 
familias tanto de la generación actual como de las generaciones futuras, por lo que el hecho de que tanto hombres y mujeres estén involucrados es la clave del éxito de este proceso. Tomando todo lo anterior como referencia, se puede confirmar una vez más, la relevancia de la participación de las mujeres en el desarrollo del país.

\section{Revisión literaria}

El empoderamiento e inclusión de la mujer es un elemento primordial en el desarrollo de las comunidades, lamentablemente no es un elemento que se tome en cuenta cuando se quiere implementar estrategias de desarrollo en una comunidad o territorio determinado y al hacerlo las mujeres en muchos casos no son participes de estas estrategias. La segregación de género en la región de América Latina y el Caribe viene marcada por elementos tales como la religión, valores culturales basados en ideas machistas y de sometimiento de la mujer, la falta de recursos económicos y los enclaves multiétnicos. Junto a ello, el empleo desprovisto y precario, el bajo acceso a recursos económicos, como la adquisición de tierras son parte de los aspectos que impiden avanzar en la reducción de las desigualdades. En América Latina no se puede entender la condición de vida de las mujeres si no se es capaz de comprender la estructura social y la falta de cohesión social en las comunidades, y de parte de los gobiernos hacia las comunidades (Ruiz-Seisdedos \& Bonometti, 2010).

A lo largo de los años se ha venido luchando en pro de la visibilidad de las mujeres, de que se alcance la equidad de género, en un sistema social donde las mujeres deben ejercer labores limitadas por su género, en el 2000 se crearon los Objetivos de Desarrollo del Milenio (ODM), los cuales fueron pactados por los 189 países miembros de las Naciones Unidas quienes acordaron cumplirlos para el 2015, dentro de estos objetivos se encuentra el promover la igualdad de género y el empoderamiento de la mujer, a finales del 2015 se realizó un análisis de los resultados de estos objetivos los cuales indican que en el área de igualdad de género y empoderamiento de la mujer:

1. El acceso a la educación de niñas ha incrementado considerablemente en comparación al año 2000.

2. Los territorios en desarrollo han alcanzado la meta de eliminar la desigualdad de géneros en la enseñanza primaria, secundaria y terciaria.

3. En Asia meridional, en el 2014 se matricularon 103 niñas por cada 100 niños.

4. Las mujeres conforman el $41 \%$ de la fuerza laboral remunerada en sectores no agrícolas, lo que significa un aumento en comparación con el 35\% de 1990.

5. Entre 1991 y 2015 el índice de mujeres en situaciones laborales vulnerables como parte del total de mujeres empleadas se redujo en un $13 \%$.

6. En los últimos 20 años la representación parlamentaria ha incrementado en casi el 90\% de los 174 países para los que se dispone de datos (F-ODM, 2012). 
Cuando hablamos directamente del desarrollo de la sociedad nicaragüense, esta ha contado con el aporte de las mujeres en sus diferentes ámbitos: económico, político, social y cultural. Sin embargo, esa contribución sólo se ha puesto de manifiesto en determinados momentos, quedando por lo regular considerablemente relevada. La presencia de las mujeres siempre fue mucho mayor en la base del sistema social que en las cúspides políticas e institucionales.

Por otra parte, la condición de las mujeres ha sufrido modificaciones en relación con la accidentada historia de Nicaragua. Tras el intento nacionalista impulsado por el General Sandino, la crisis nicaragüense provocó el establecimiento de una dictadura familiar (1933 y 1979) que fue desalojada del poder por una revolución armada. La participación de las mujeres en la lucha por derrocar la dictadura de los Somoza y el aumento notable de su presencia durante el intenso proceso sociopolítico de los años ochenta, si bien no cambió por completo la tendencia histórica de su reducida presencia en los espacios de toma de decisión, mejoró la presencia de las mismas provocando un salto en la visibilidad de las mujeres.

Uno de los cambios importantes que introdujo esa crisis fue la fuerte aceleración de la tendencia a una mayor participación económica femenina, especialmente en términos comparativos con el varón, dado que una alta proporción de los hombres jóvenes se incorporaron a las filas militares. Desde mediados de los años ochenta se registra que las mujeres son más de un tercio de la fuerza laboral del país. Aunque ese aumento de la participación femenina significo que las mujeres accedieran a profesiones tradicionalmente consideradas como masculinas, todavía se concentraban principalmente en los servicios personales o como empleadas de oficina, teniendo una baja participación en el sector productivo. A pesar de los cambios que se produjeron en 1990 con el cambio de gobierno, las mujeres siguieron estando ocultas o al menos las acciones que estas realizaban no se veía como algo relevante, después del 2007 con el traspaso de gobierno se inició un conjunto de cambios en las políticas sociales para hacer más visible el papel de las mujeres en el desarrollo del país.

Actualmente según lo describe la Sra. Silvia Rucks, representante residente del PNUD, Nicaragua muestra importantes avances en materia de género en varios de los ODMs: reducción de la pobreza extrema, de la desnutrición crónica y de la mortalidad materna son algunos ejemplos. Nicaragua es uno de los países que tiene el mayor porcentaje de mujeres en cargos públicos. Destaca de manera particular la participación de $42 \%$ de mujeres en la Asamblea Nacional. Las mujeres además superan en todos los niveles educativos la matrícula de los varones, tienen mejores índices de retención escolar y mejores calificaciones. Y esos avances también empiezan a ser reconocidos a nivel internacional; recientemente el PNUD de Nicaragua recibió la certificación del Sello de Oro por la Igualdad de Género, que no es más que el reconocimiento al progreso alcanzado en el país en materia de género, fruto del trabajo articulado de los diversos sectores nacionales, del PNUD y los socios de la cooperación internacional. 
Pero en el ámbito económico las mujeres de Nicaragua enfrentan desigualdades, principalmente por las condiciones y características de su inserción laboral. El Reporte Global de Equidad de Género ubica a Nicaragua en noveno sitio en igualdad de oportunidades para las mujeres, convirtiéndolo en el mejor país de Centroamérica en esta materia (Rucks, 2015). En Nicaragua existen un sinnúmero de casos que nos permiten corroborar que la promoción del empoderamiento de las mujeres, permite a estas configurar sus vidas de manera positiva, permitiéndoles tomar el control sobre todos aquellos elementos que afectan su desarrollo ya sea político, social, cultural o económico. A continuación, se mencionan tres casos como ejemplo vivo del cambio que se puede generar en las mujeres, sus familias y la comunidad a través del empoderamiento e inclusión de la mujer.

\section{a) Red de mujeres chontaleñas: "Empoderamiento Político Organizativo"}

La fundación para el desarrollo de las mujeres chontaleñas, es parte integral de las Red de Mujeres Chontaleñas, esta es una asociación civil sin fines de lucro que funciona como tal desde el 2010. Esta es una organización establecida como Red, que trabaja en pro de la formación integral y para el empoderamiento de las mujeres. Uno de sus logros es el hecho de que las directivas de la Red han adquirido la capacidad de generar sus propios ingresos con actividades que no perjudiquen a los otros grupos integrantes de la Red, con el fin de dejar de depender de la cooperación externa.

Por otra parte, realizaron una autoevaluación y una de las principales lecciones aprendidas en el proceso de alineación, disposición y fortalecimiento de la Red de Mujeres Chontaleñas es el hecho de que para las mujeres no puede haber desarrollo local, en ninguna de sus dimensiones si en ello no se plantea el tema de género y empoderamiento de la mujer. Pero, también en el proceso han comprendido que la integración del enfoque de género en el quehacer de su organización requiere de la comunicación y el trabajo en equipo de todos los actores involucrados. Las integrantes de la Red están sumidas en el proceso de ordenamiento de la organización, autoevaluando los adelantos de la Red y atendiendo el crecimiento. Además, han sabido emplear de manera efectiva la política de alianzas con otros actores, con el objetivo de fortalecer y mejorar la Red para seguir con el crecimiento de la misma. Sin embargo, se ha avanzado mucho con pocos recursos, se ha potenciado al máximo lo que se tenía y se ha logrado dar visibilidad a la Red en cada territorio.

\section{b) Finca esperanza verde turismo comunitario (San Ramón-Matagalpa)}

En el municipio de San Ramón-Matagalpa se encuentra la finca esperanza verde, fue creada con el fin de fortalecer los lazos de cooperación entre en municipio y una ONG de origen norteamericano, esta iniciativa consistió en una casa de huéspedes manejada por un grupo de 7 mujeres, las cuales alojaban a los turistas en sus propias 
casas, para que estos vivieran la realidad de estas mujeres a través de la convivencia con las familias (Alarcon \& Ferguson, 2011).

Uno de los resultados más importantes es que les permitió a las mujeres tener un ingreso económico y a la vez comenzar con el hábito del ahorro, que les permite mejorar sus condiciones de vida, como por ejemplo poder pagar servicios médicos privados. Por otra parte, muchas de las mujeres mencionaban que no conocían cuales eran sus derechos y qué leyes, decretos o normativas municipales eran de beneficio para ellas; sin embargo, desde que se integraron a la iniciativa dicen conocer ahora muy bien sus derechos fundamentales. Además, esta iniciativa dejo de ser sólo para mujeres, si no que les ha permitido a las participantes realizar un trabajo colaborativo entre los integrantes de las familias. Esta iniciativa abrió puertas para que las mujeres participen de manera activa en reuniones del consejo municipal, además de prepararlas en áreas como primeros auxilios y turismo comunitario.

\section{c) Eco-albergue turístico entre brisas y encantos. (La Fundadora-Jinotega)}

El eco-albergue turísticos entre brisas y encantos surge como resultados de las estrategias de la Cooperativa de mujeres y coordinada por la ONG La Cuculmeca ubicada en Jinotega, esta iniciativa comenzó en el 2010, en donde en ese momento las mujeres participantes no percibían ningún tipo de remuneración económica, ya que todo el dinero obtenido del albergue era reinvertido. Sin embargo, esta iniciativa a pesar de que no se percibió dinero se trataba de un empleo decente, además de ser partícipes de un proceso que sería punta de lanza para el desarrollo de esta comunidad. Actualmente las mujeres ya perciben salarios que les permiten tener recursos económicos de los que antes no disponían (Alarcon \& Ferguson, 2011). Actualmente las mujeres participan en la esfera pública de su comunidad, son parte de los actualmente denominados consejos de la familia y la comunidad, aproximadamente más del 40\% de las mujeres de la comunidad han abierto negocios vinculados al turismo comunitario y realizan un trabajo colaborativo con los integrantes de su familia.

\section{Diseño metodológico}

El estudio fue descriptivo de corte transversal, por lo que se da la descripción de un fenómeno en el período de octubre a diciembre del 2016. El universo de la investigación era la población total de la comunidad de Tonalá que es de 4,645 habitantes. Del universo se tomó la población económicamente activa de la comunidad que corresponde a 1,237 habitantes según datos del Instituto Nacional de Información del Desarrollo (INIDE, 2012), las cuales oscilan entre los 20 y 40 años. Población y muestreo de la investigación. La población total fueron las mujeres económicamente activas en la comunidad de Tonalá, que era de 1,237 habitantes. 
Una vez que se definió la población, se aplicó el método estadístico STATS, versión 2.o para obtener la muestra, que en este caso fue de 293 pobladores. El objeto de estudio de esta investigación fueron las mujeres de la comunidad de Tonalá. Plan de recolección, manejo y análisis de la información. Una vez elaborado el instrumento se realizaron dos viajes cada semana durante el período de estudio (octubre a diciembre 2016) a la comunidad de Tonalá para la recolección de los datos, donde se aplicaron las encuestas que fueron distribuidas equitativamente en los 12 barrios de la comunidad de Tonalá, resultando aproximadamente 24 encuestas por barrio. Los resultados obtenidos se procesaron a través de paquete estadístico IBM SPSS Statistics 18 y se presentaron a través de tablas y gráficos.

\section{Resultados}

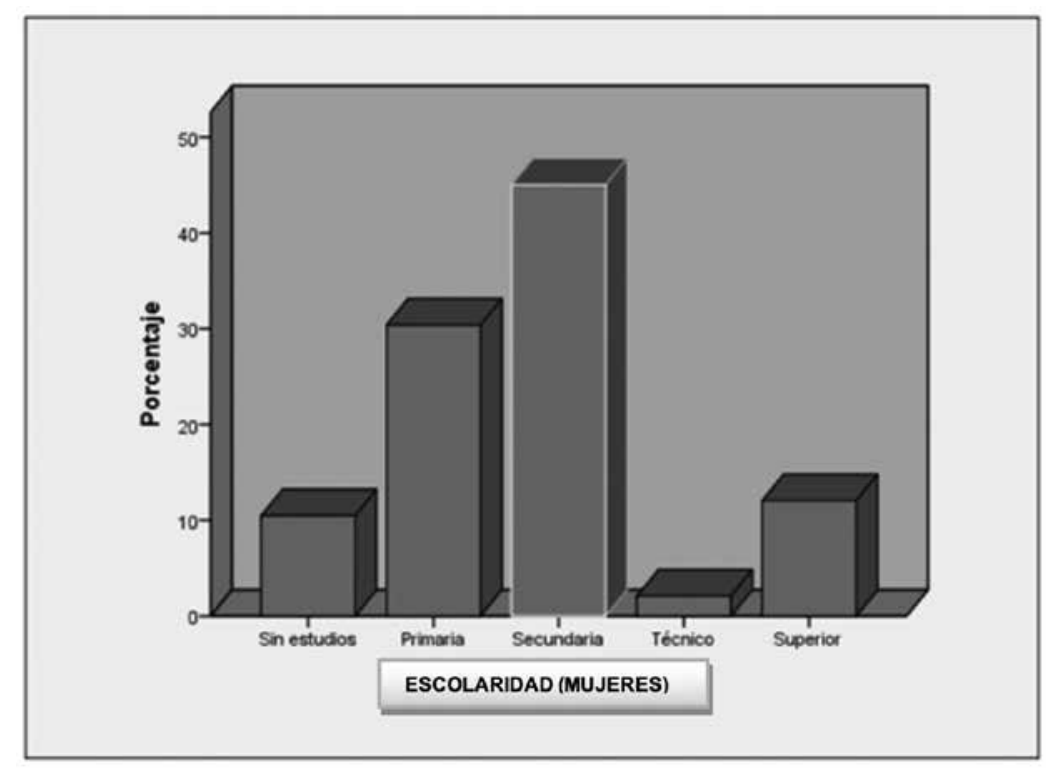

Figura 1: Escolaridad de las Mujeres

$\mathrm{El} 30 \%$ de las mujeres encuestadas concluyo sus estudios primarios y $50 \%$ de ellas lograron culminar la secundaria, sólo un 12\% tiene estudios superiores, un $4 \%$ de ellas tienen estudios técnicos, un 10\% de ellas no tienen ningún tipo de estudio. 


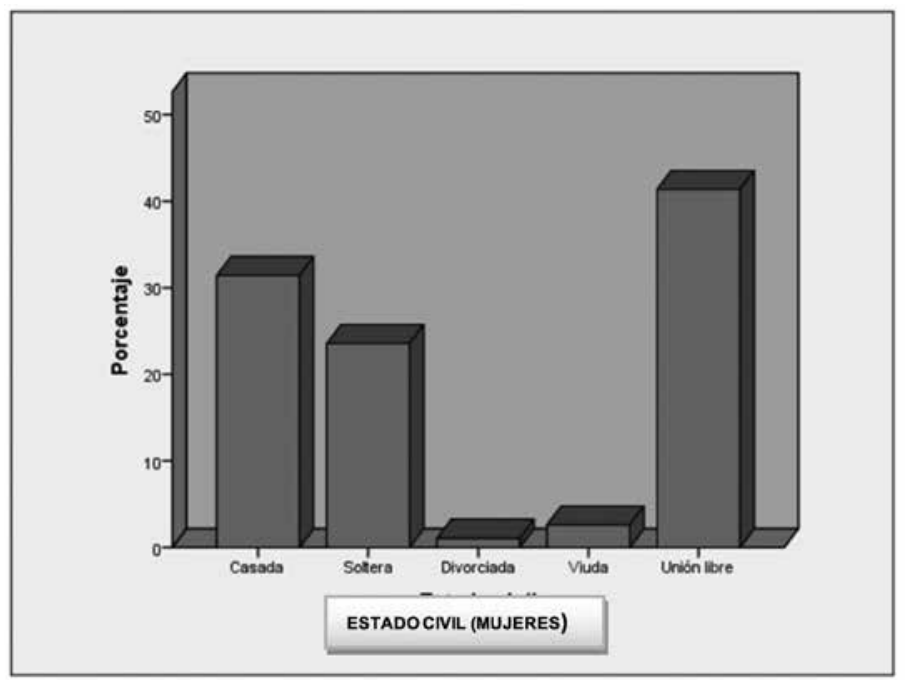

Figura 2: Estado civil de las mujeres

De las mujeres encuestadas el 43\% están en unión libre y solo el 34\% de ellas están casadas, un $26 \%$ están solteras y por debajo del $5 \%$ están divorciadas o quedaron viudas.

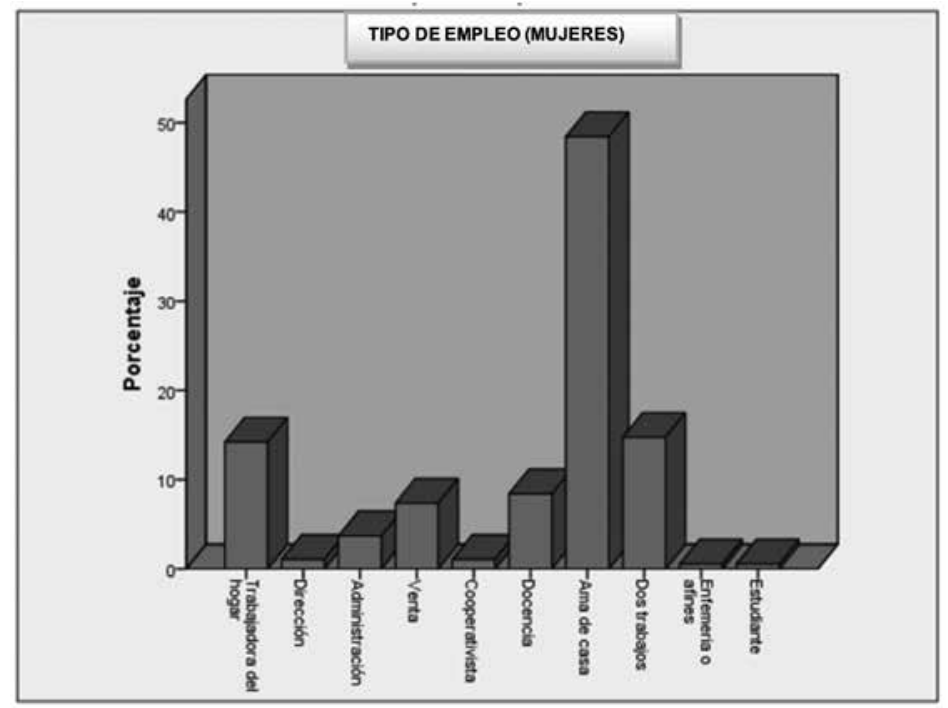

Figura 3: Tipo de empleo que ejercen las mujeres

El 50\% de estas mujeres es ama de casa y el $18 \%$ ejercen dos trabajos o son asistentes del hogar y el otro $32 \%$ realizan diferentes actividades. 


\section{GÉNERO E INTERCULTURALIDAD}

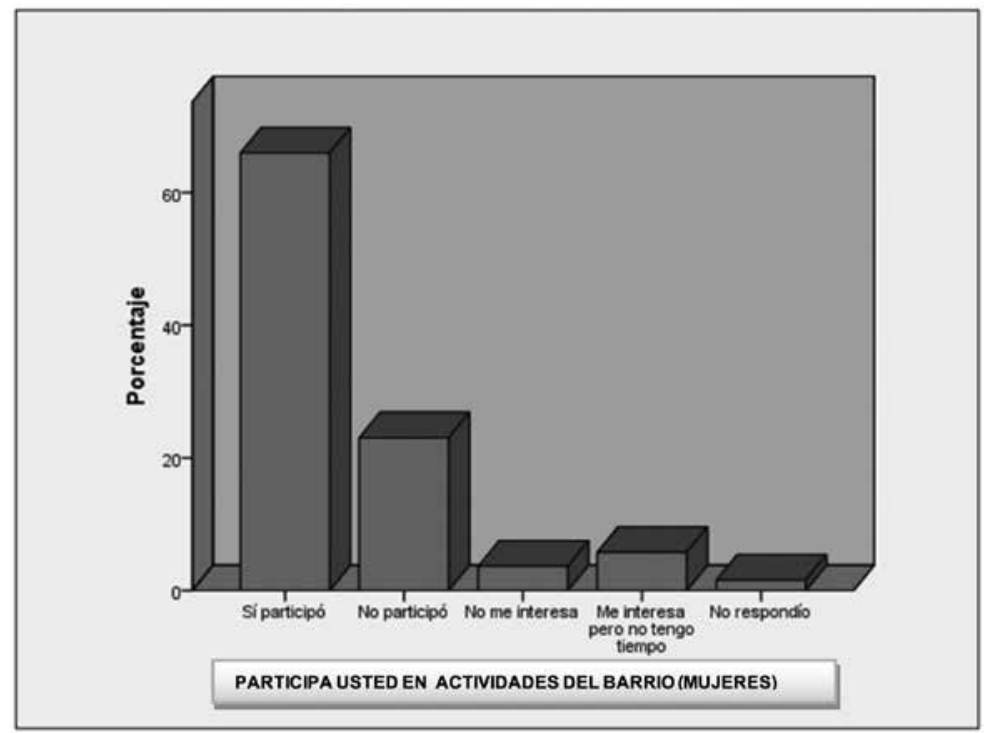

Figura 4: ¿Participa usted en actividades del barrio (Mujeres)?

El 62\% de las mujeres respondieron que sí participan, mientras el 21\% respondieron que no participaban, el restante $17 \%$ se divide entre aquellas mujeres que no le interesa participar, no tienen tiempo o bien no respondieron. (Ver justificación en el cuadro 1)

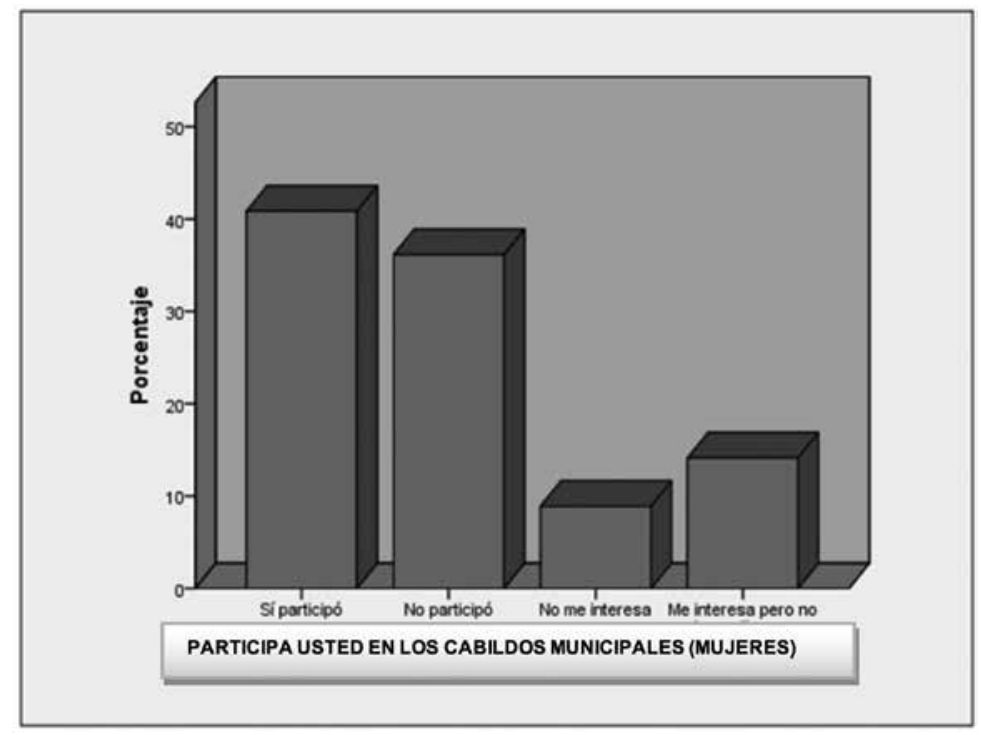

Figura 5: ¿Participa usted en los cabildos municipales (Mujeres)?

El 42\% de las mujeres respondieron que sí participaban, mientras el 38\% dijo no hacerlo, un $13 \%$ dijo no tener tiempo y un $7 \%$ dijo no interesarle (Ver justificación cuadro 2). 


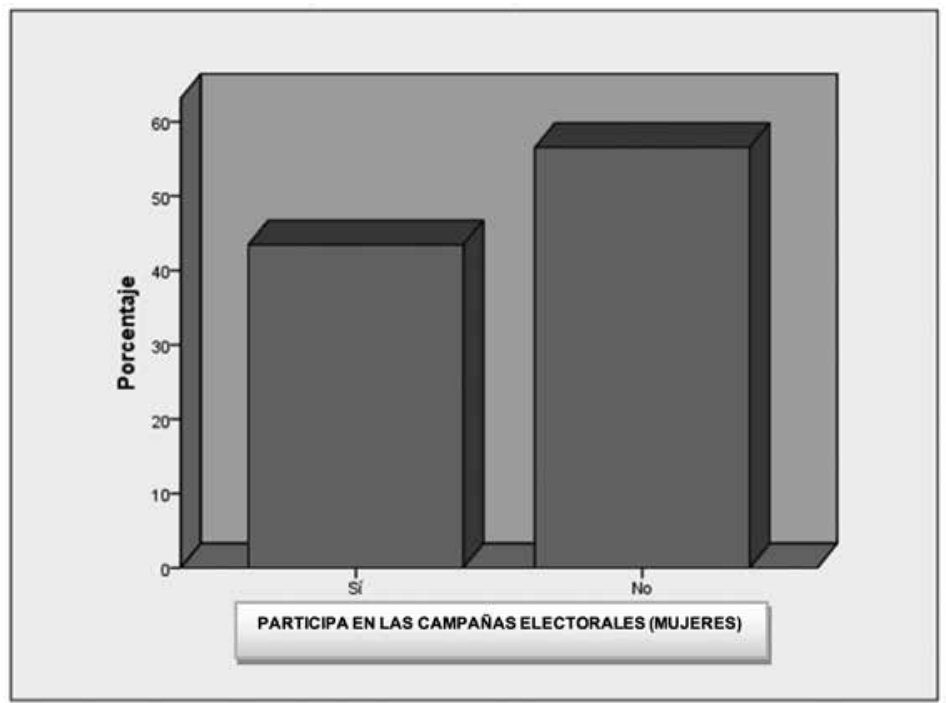

Figura 6: ¿Participa en las campañas electorales (Mujeres)?

El 42\% de las mujeres respondieron que sí participaban, mientras el 58\% dijo no hacerlo. (Ver justificación en cuadro $\mathrm{N}^{\circ} 3$ )

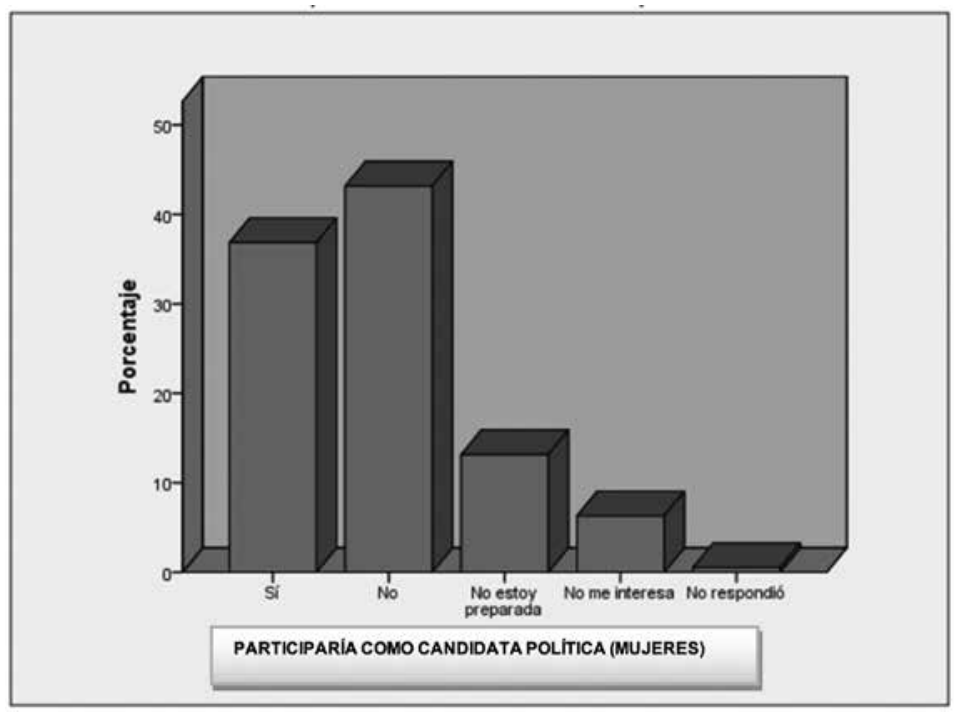

Figura 7: Participación politica de las mujeres

$\mathrm{El} 45 \%$ de las mujeres respondieron que no participaban, mientras el $37 \%$ dijo que si lo haría, un $11 \%$ dijo no sentirse preparada, un $6 \%$ dijo no interesarle y un $1 \%$ no respondió (Ver justificación en cuadro 4). 


\section{GÉNERO E INTERCULTURALIDAD}

Cuadro 16: Causas de la falta de participación de las mujeres en las actividades del barrio y la comunidad.

\begin{tabular}{|l|l|l|}
\hline \multicolumn{1}{|c|}{ ¿Por qué no participa en las actividades del barrio? (Mujeres) } & Frecuencia & Porcentaje \\
\hline Dirigentes & 2 & 3.9 \\
\hline Falta de apoyo & 2 & 3.9 \\
\hline Falta de equidad & 3 & 5.9 \\
\hline No invitan & 10 & 19.6 \\
\hline No me gusta & 2 & 3.9 \\
\hline No respondió & 32 & 62.7 \\
\hline Total & 51 & 100.0 \\
\hline
\end{tabular}

Cuadro 2: Razones por las cuales las mujeres no participan en los cabildos municipales.

\begin{tabular}{|l|l|l|}
\hline \multicolumn{1}{|c|}{ ¿Por qué no participa en cabildos municipales? (Mujeres) } & Frecuencia & Porcentaje \\
\hline Dirigente & 4 & 5.0 \\
\hline Falta de beneficios & 1 & 1.3 \\
\hline Inasistencia & 1 & 1.3 \\
\hline No es importante & 1 & 1.3 \\
\hline No invitan & 1 & 1.3 \\
\hline No me gusta & 2 & 2.5 \\
\hline No respondió & 66 & 82.5 \\
\hline No sabe & 2 & 2.5 \\
\hline Pérdida de tiempo & 1 & 1.3 \\
\hline Religión & & \\
\hline Total & 80 & 100 \\
\hline
\end{tabular}

Cuadro 3: Razones por las cuales las mujeres no participan en las campañas electorales nacionales y municipales.

\begin{tabular}{|l|l|l|}
\hline ¿Por qué no participa en las campañas electorales? (Mujeres) & Frecuencia & Porcentaje \\
\hline Enfermedad crónica & 1 & .9 \\
\hline Falta de tiempo & 20 & 18.3 \\
\hline Indocto & 1 & .9 \\
\hline Inseguridad & 3 & 2.8 \\
\hline No cumplen & 2 & 1.8 \\
\hline No invitan & 11 & 10.0 \\
\hline No me gusta & 27 & 24.8 \\
\hline No respondió & 33 & 30.3 \\
\hline No sabe & 2 & 1.8 \\
\hline
\end{tabular}




\begin{tabular}{|l|l|l|}
\hline ¿Por qué no participa en las campañas electorales? (Mujeres) & Frecuencia & \multicolumn{1}{c|}{ Porcentaje } \\
\hline Pérdida de tiempo & 3 & 2.8 \\
\hline Porque no & 1 & .9 \\
\hline Religión & 6 & 5.5 \\
\hline Total & 109 & 100.0 \\
\hline
\end{tabular}

Cuadro 4: Razones por las cuales las mujeres no participarían como candidata política.

\begin{tabular}{|l|l|l|}
\hline \multicolumn{1}{|c|}{ ¿Por qué no participaría como candidata política? (Mujeres) } & Frecuencia & Porcentaje \\
\hline Es por vocación & 2 & 1.6 \\
\hline Falta de tiempo & 5 & 4.1 \\
\hline Genera problemas & 6 & 4.9 \\
\hline Indocto & 1 & .8 \\
\hline No darían oportunidad & 3 & 2.4 \\
\hline No me gusta & 9 & 7.3 \\
\hline No me siento capaz & 2 & 1.6 \\
\hline No respondió & 95 & 77.2 \\
\hline Total & 123 & 100.0 \\
\hline
\end{tabular}

Cuadro 5: ¿Cómo consideran los hombres la participación de las mujeres en la comunidad?

\begin{tabular}{|c|c|c|c|c|}
\hline Muy Importante & Importante & Poco importante & Sin importancia & No respondió \\
\hline $60 \%$ & $18 \%$ & $14 \%$ & $8 \%$ & $0 \%$ \\
\hline
\end{tabular}

\section{Conclusiones}

Cuando se habla de participación se piensa primera en la disponibilidad de tiempo, en cuanto al empleo de las mujeres los datos indican que la mitad de la población encuestada es ama de casa y aunque este es uno de los trabajos que demanda mayor tiempo, esfuerzo y no se obtiene remuneración alguna, realizar esta actividad les impide a las mujeres desarrollarse en otras áreas, dentro y fuera de su familia y comunidad.

La Constitución Política de Nicaragua enseña que el acceso a la educación, empleo y servicios básicos son derechos de todos y cada uno de los nicaragüenses; sin embargo, la participación también es parte de los derechos y es una de las características de un gobierno democrático como el de Nicaragua. De los encuestados más de la mitad de la población indicó que sí participaba, al menos en una de las actividades realizadas dentro de su comunidad, lo que es un indicador positivo en temas de participación, a pesar de ello se mantiene un 40\% de la población que no participa por muchas razones, alegando principalmente estar en desacuerdo con los dirigentes políticos del barrio porque "no les gusta" o no le ven ninguna importancia a las actividades, ya que muchos de ellos ven estas actividades como protocolo para hacer al final lo que a los dirigentes 
les parezca mejor, por otro lado, la Alcaldía Municipal y la Policía Nacional indicaban que dentro de sus planes de acción está el fomentar la participación ciudadana.

En el caso de los cabildos municipales, se presenta una situación asociada a lo antes expuesto, ya que los resultados obtenidos indican que la participación femenina no sobrepasa el 50\% de participación, en muchas ocasiones, por el desacuerdo con el dirigente a cargo de llevar acabo esta actividad. Los cabildos municipales aparte de ser un método de participación establecido en la Constitución Política de Nicaragua, es también una técnica efectiva que les permite a las autoridades locales conocer de primera mano las necesidades de la población, lo que fomentaría la dimensión política del desarrollo local, en donde una de sus bases fundamentales es la participación ciudadana.

Es importante recalcar que el método de participación se efectúa por parte de las autoridades y es responsabilidad de los pobladores asistir, si quieren ejercer cambios dentro de su comunidad, no puede haber cambios sin participación. Sin embargo, el porcentaje que dijo estar dispuesto a participar como candidato político no supera el $42 \%$, lo que hace notar como bajan los índices de participación entre más pública se vuelve esta actividad. En muchas ocasiones, esto es debido, no a la falta de capacidad, sino a la falta de confianza de los ciudadanos, argumentan que no participan porque no los toman en cuenta, o por evitar problemas, o bien porque no les gusta, muchas veces limitan su participación por temor a equivocarse, porque se les ha enseñado que fallar sólo lo hacen las personas sin capacidades, pero esos comentarios son parte de la cultura que debe cambiarse, por tanto para incrementar el empoderamiento e inclusión de la mujer dentro de la comunidad, se debe trabajar en la dimensión cultural del desarrollo pues esto significa un cambio de mentalidad que no se puede hacer a corto plazo, sino que es un proceso paulatino.

Por otro lado, el tipo de empleo de las mujeres está relacionado con su participación en las elecciones y lo más relevante es que quienes votan son las amas de casa y las trabajadoras del hogar. A pesar de lo antes mencionado, las amas de casa que no participan indicaba que se debe a falta de tiempo o porque simplemente no quieren. Además, la falta de participación podría deberse a que la ama de casa siente que su opinión no tiene valor alguno, lo alarmante es que esta es una situación que no solo sucede en las mujeres que son amas de casa, sino en aquellas que gozan de un trabajo formal, estudios superiores y en donde se supone deberían tener mayor poder de decisión.

En definitiva, a pesar de que se está promoviendo la participación femenina como técnica de inclusión a nivel nacional, las mujeres de la comunidad de Tonalá siguen sin ejercer con creces ese derecho, según los censos realizados por INIDE, indican que la densidad poblacional femenina está casi igualada a la densidad poblacional masculina con una mínima diferencia a favor de las mujeres, por ello la participación 
equitativa es de suma importancia para el desarrollo local, a pesar de esto las mujeres de la comunidad de Tonalá no participan, y entre más pública sea la actividad en la que participan, menor es su participación, todo esto lleva a pensar que si las mujeres se decidieran a participar en todas las actividades que afectan directa o indirectamente sus vidas y las de su familia, serían capaces de generar grandes cambios en el país y en su comunidad.

\section{Lista de referencias}

Alarcon, D. M., \& Ferguson, L. (2011). El turismo como oportunidad. Cyan, Proyectos Editoriales, S.A.

Bou, M. (2003). La participación de las mujeres en la política. El caso de América Latina. Recuperado de http://iknowpolitics.org/sites/default/files/doc_74_la-participacion-de-las-mujeres-en-la-politica.pdf

Bareiro, Line, Soto, Clyde, Soto y Lilian. (2007). La inclusión de las mujeres en los procesos de reforma política en América Latina. Recuperado de https://publications. iadb.org/handle/11319/5044?locale ttribute $=$ pt\&scope $=123456789 / 6 \&$ thumb nail=false\&order $=$ desc\&rpp $=5 \&$ sort $\_b y=$ score\&page $=0 \& q u e r y=$ genero\&gro up_by=none\&etal=o

Bock, G. (2001). La mujer en la historia de Europa. De la Edad Media a nuestros días. trad. T. de Lozoya, Crítica, Barcelona. Recuperado de: https://alpha.sib.uc.pt/?q=content/ la-mujer-en-la-historia-de-europa-de-la-edad-media-nuestros-d\% $\mathrm{C}_{3} \% \mathrm{AD}$

Bravo, O. (2007). La dimensión política del desarrollo local. Recuperado de http://www. redalyc.org/pdf/1990/199016808002.pdf .

Bareiro y Torres. (2009). El camino hacia la paridad: evaluación de las cuotas de participación política de las mujeres en América Latina. Recuperado de https://dialnet. unirioja.es/servlet/articulo?codigo $=3654973$

Duran, A. (2008). La Lucha por el voto de la mujer en EE.UU. Recuperado de: http://www. contactomagazine.com/articulos/elvotodelamujer10o8.htm\#.WV55SYSGPIU

F-ODM. (2012). Igualdad de género y empoderamiento de la mujer. Recuperado de: http:// www.un.org/sustainabledevelopment/es/gender-equality/

Jütting, J. (2012). Perspectivas de cohesión social: el pegamento que mantiene unida a la sociedad. Recuperado: http://www.un.org/es/development/desa/news/ policy/perspectivas-de-cohesion-social-el-pegamento-que-mantiene-unidaa-la-sociedad.html. 
LAZARO CASTELLANOS, R., \& ZAPATA MARTELO, E. y. (2007). Jefas de hogar: cambios en el trabajo y en las relaciones de poder. Recuperado http://www.scielo.org.mx/ scielo.php?script=sci_arttext\&pid=So188-77422007000200009.

Molina Vega, C. P. (2015). Participación política. Recuperado de: http://www.mercaba. org/FICHAS/Capel/participacion_politica.htm.

Asamblea Nacional de Nicaragua. (2007). Constitución Política de Nicaragua. Recuperada de: http://www.ineter.gob.ni/Constitucion\%2oPolitica\%20de\%20Nicargua.pdf.

Asamblea Nacional de Nicaragua. (2013). Datos estadísticos sobre igualdad y equidad de género en Nicaragua 2013. Recuperado de: http://www.asamblea.gob.ni/ ugenero/estadisticas-sobre-igualdad-y-equidad-de-genero-en-nicaragua.pdf

Asamblea Nacional de Nicaragua. (2008). Ley de igualdad y oportunidades. Recuperado de: http://legislacion.asamblea.gob.ni/Normaweb.nsf/(\$All)/DFACDD675534 DACE0625744Boo77C73F?OpenDocument.

Ríos- Molina, L. M., \& Rivas, J. A. (2016). Mujeres y participación política en Nicaragua, 1980 -2015. Recuperado http://revista.unanleon.edu.ni/index.php/REBICAMCLI/ article/view/168.

Rohland, H. v. (2010). Historia. Barcelona, España, Editorial desconocida, 3 educación del $2010 \mathrm{~N}^{\circ} 70$ Pag.5.

Rucks, S. (2015). El mercado laboral de Nicaragua desde un enfoque de género. Recuperado de: http://www.ni.undp.org/content/nicaragua/es/home/presscenter/speeches/2015/o7/14/el-mercado-laboral-de-nicaragua-desde-un-enfoque-de-gnero.html.

Ruiz Seisdedos, S., \& Bonometti, P. (2010). Las mujeres en América Latina: indicadores $y$ datos. Recuperado de http://unpan1.un.org/intradoc/groups/public/documents/icap/unpano46955.pdf.

Rivas, Y. W., Salgado, O., Narvaéz, E., \& León, L. (2015). Propuesta de plan estratégico para la mejor utilización del sub-programa de gobieno "Bono productivo alimentario". Recuperado de: http://riul.unanleon.edu.ni:8o8o/jspui/bitstream/123456789/4973/1/230583.pdf. 